\title{
Comparison of clinical characteristics between patients with coronavirus disease 2019 (COVID-19) who retested RT-PCR positive versus negative: a retrospective study of data from Nanjing
}

\author{
Yuan Liu ${ }^{1 \#}$, Ning Ding ${ }^{2 \#}$, Shixiang Zhou ${ }^{3}$, Chen Chen ${ }^{1}$, Shan Huang ${ }^{1}$, Yanling Lv $^{4}$, Damin Jiao ${ }^{5}$, \\ Yishan Zheng ${ }^{6}$, Zhiliang $\mathrm{Hu}^{1}$, Chuanjun $\mathrm{Xu}^{7}$, Wei Chen ${ }^{8}$, Hui Dai ${ }^{9}$, Wenkui Sun ${ }^{2}$, Cong Cheng ${ }^{1}, \mathrm{Ru} \mathrm{Lv}^{1}$, \\ Jian Cheng ${ }^{1}, \mathrm{Zi} \mathrm{Ye}{ }^{1}$, Zhengjie $\mathrm{Li}^{1}$, Yongxiang $\mathrm{Yi}^{10}$, Hongxia Wei ${ }^{1}$ \\ ${ }^{1}$ Department of Infectious Disease, The Second Hospital of Nanjing, Nanjing University of Chinese Medicine, Nanjing, China; ${ }^{2}$ Department of \\ Respirology and Critical Care Medicine, The First Affiliated Hospital of Nanjing Medical University; Nanjing, China; ${ }^{3}$ Department of osteology, \\ The Second Hospital of Nanjing, Nanjing University of Chinese Medicine, Nanjing, China; ${ }^{4}$ Department of Respirology and Critical Care Medicine, \\ The Second Hospital of Nanjing, Nanjing University of Chinese Medicine, Nanjing, China; ${ }^{5}$ Department of Rheumatology and Immunology, The \\ Second Hospital of Nanjing, Nanjing University of Chinese Medicine, Nanjing, China; ${ }^{6}$ Intensive Care Unit, The Second Hospital of Nanjing, \\ Nanjing University of Chinese Medicine, Nanjing, China; ${ }^{7}$ Department of radiology, The Second Hospital of Nanjing, Nanjing University of \\ Chinese Medicine, Nanjing, China; ${ }^{8}$ Department of laboratory, The Second Hospital of Nanjing, Nanjing University of Chinese Medicine, Nanjing, \\ China; ${ }^{9}$ Department of Medical Records Statistics, The First Affiliated Hospital of Nanjing Medical University; Nanjing, China; ${ }^{10}$ Department of \\ Hepatobiliary Surgery, The Second Hospital of Nanjing, Nanjing University of Chinese Medicine, Nanjing, China \\ Contributions: (I) Conception and design: Y Liu, N Ding, Y Yi, H Wei; (II) Administrative support: Y Yi, H Wei; (III) Provision of study materials \\ or patients: None; (IV) Collection and assembly of data: Y Liu, S Zhou, C Chen, S Huang, Y Lv, D Jiao, Y Zheng, Z Hu, C Xu, W Chen, H Dai, \\ W Sun, C Cheng, R Lv, J Cheng, Z Ye, Z Li; (V) Data analysis and interpretation: N Ding, H Dai; (VI) Manuscript writing: All authors; (VII) Final \\ approval of manuscript: All authors. \\ "These authors contributed equally to this work. \\ Correspondence to: Hongxia Wei, MD. Department of Infectious Disease, Second Hospital of Nanjing, Nanjing University of Chinese Medicine, \\ 1-1 Zhongfu Road, Nanjing 210003, China. Email: njyy019@njucm.edu; Yongxiang Yi, MD. Department of Hepatobiliary Surgery, Nanjing \\ infectious Disease Center, The Second Hospital of Nanjing, Nanjing University of Chinese Medicine, 1-1 Zhongfu Road, Nanjing 210003, China. \\ Email: ian0126@126.com.
}

Background: The epidemiological and clinical characteristics of patients with coronavirus disease 2019 (COVID-19) have been reported. However, the prevalence of retesting positive by RT-PCR for the severe acute respiratory syndrome coronavirus 2 (SARS-CoV-2), and the associated patient characteristics, remain unclear.

Methods: We included 90 confirmed cases of COVID-19 treated in the Nanjing Public Health Center from January 20, 2020 to February 16, 2020 in this retrospective study. All patients completed treatment for COVID-19 and were retested by RT-PCR for SARS-CoV-2 4-20 days after completion of therapy. The clinical characteristics between patients with who retested positive versus negative by RT-PCR were compared, and the factors predictive of positive retesting were analyzed. Positive retesting was modeled with the area under the receiver operating characteristic curve (AUC).

Results: The age range of the study population was 0.8-97 years, and all patients were cured or showed improvement. A total of 10 (11\%) patients retested positive by RT-PCR 4-20 days after completion of therapy. As compared with patients who retested negative, those who retested positive had a lower percentage of pre-admission fever, a higher percentage of post-admission fever, a lower percentage of bilateral lung infection, higher white blood cell (WBC) count and creatine phosphokinase, and lower hypersensitive c-reactive protein (hs-CRP), interleukin-6 and erythrocyte sedimentation rates (all $\mathrm{P}<0.05$ ). Logistic regression analysis of the above eight key variables showed that lower hs-CRP and higher WBC 
were independently associated with positive retesting by RT-PCR. A combination of hs-CRP and WBC were predictive of positive retesting, with an AUC of 0.859 .

Conclusions: Patients with COVID-19 who retested positive by RT-PCR for SARS-CoV-2 had mild symptoms and better blood testing results. A combination of hs-CRP and WBC may predict positive retesting by RT-PCR; however, the sensitivity and specificity should be studied further.

Keywords: Coronavirus disease 2019 (COVID-19); severe acute respiratory syndrome coronavirus 2 (SARSCoV-2); re-positive

Submitted Apr 09, 2020. Accepted for publication Aug 13, 2020.

doi: $10.21037 /$ jtd.2020.04.17

View this article at: http://dx.doi.org/10.21037/jtd.2020.04.17

\section{Introduction}

In December 2019, a series of pneumonia cases of unknown cause emerged in Wuhan, Hubei, China $(1,2)$. Deep sequencing analysis of lower respiratory tract samples indicated a novel coronavirus, which was named severe acute respiratory syndrome coronavirus 2 (SARS-CoV-2) (3). As of 5 March the number of confirmed cases of coronavirus disease 2019 (COVID-19) worldwide surpassed 96,000 , and the number of deaths was more than 3,300 (4). The epidemiological and clinical characteristics, and the imaging features of COVID-19 have been well described (5,6). Lan et al. (7) has reported that patients who have recovered from COVID-19 may test positive by a real-time reversetranscriptase-polymerasechain-reaction (RT-PCR); however, the characteristics and risk factors of people who retest positive for SARS-CoV-2 by RT-PCR (denoted re-positive patients) remain unclear. Here, we present a detailed comparison of clinical features between re-positive patients and those who retest negative by RT-PCR (denoted negative patient) after therapy, according to data from Nanjing.

\section{Methods}

\section{Patients}

This case series was approved by the Institutional Ethics Board of the Second Hospital of Nanjing (No. 2020-L-Sky003). Oral consent was obtained from patients. All patients who were admitted to Nanjing Public Health Medical Center between January 20, 2020 and February 16, 2020 and confirmed to have COVID-19, according to the diagnostic and treatment guidelines for SARS$\mathrm{CoV}-2$ issued by the Chinese National Health Committee (version 3-6), were included in this study. Pharyngeal swab specimens were collected from these patients, and SARSCoV-2 detection was performed. Confirmed COVID-19 cases were defined by a positive result in high-throughput sequencing or a RT-PCR assay of nasal and pharyngeal swab specimens. All patients were re-tested by RT-PCR for 2019-nCoVSARS-CoV-2 4-20 days after completion of therapy. The criteria for hospital discharge in China were normal temperature for a period longer than 72 hours, absence of clinical symptoms and radiological abnormalities, and two negative RT-PCR test results separated by at least 48 hours. Patients with COVID-19 who met the criteria were able to be discharged. However, all patients were required to receive a third RT-PCR test before discharge. If the test was negative, the patient was discharged; if the test was positive (referred to as re-positive), the patient was not discharged but remained in the hospital for 2 weeks of additional medical observation. The re-positive patients received antiviral treatment (according to the diagnostic and treatment guidelines for SARS-CoV-2 issued by the Chinese National Health Committee, version 6) and a throat swab novel coronavirus nucleic acid was tested every 24-48 hours. Antiviral treatment was terminated when the patient has three consecutive negatives of retesting RTPCR of SARS-CoV-2.

\section{Data collection}

We obtained the medical records and compiled data for hospitalized patients with laboratory-confirmed COVID-19, between January 20, 2020 and March 17, 2020. The data cutoff for the study was March 18, 2020. We extracted demographic data, medical history, recent exposure history, underlying comorbidities, clinical symptoms or signs, laboratory findings on admission, chest computed tomographic (CT) scans, results of throat swab 
novel coronavirus nucleic acid tests and treatment measures (including antiviral therapy, corticosteroid therapy and respiratory support) from electronic medical records.

The date of disease onset was that on which symptoms were noticed. Exposure history was defined as exposure to people with confirmed SARS-CoV-2 infection or to Wuhan and the surrounding area within 14 days of illness onset. The number of days between exposure and symptom onset was defined as the incubation period. CT and all laboratory tests were performed according to the clinical care needs of the patients. We determined the presence of radiologic abnormalities based on the documentation or description in medical charts; if imaging scans were available, they were reviewed by two respiratory medicine attending physicians who extracted the data. Major disagreements between the two reviewers were resolved by consultation with a senior reviewer. Laboratory assessments consisted of a complete blood count and lymphocyte classification count, blood chemical analysis, arterial blood gas, coagulation testing, assessment of liver and renal function, and measures of blood sedimentation, hypersensitive C-reactive protein (hsCRP), procalcitonin, lactate dehydrogenase, creatine kinase, interleukin-6 (IL-6) and erythrocyte sedimentation rate (ESR). We defined the degree of severity of COVID-19 at the time of admission by using the diagnostic and treatment guidelines for SARS-CoV-2 issued by the Chinese National Health Committee for community-acquired pneumonia (version 5-6). Data were entered into a computerized database and cross-checked. All data were collected and checked by three physicians (S Zhou, C Chen and S Huang).

\section{Statistical analysis}

All continuous variables were distributed non-normally and are presented as medians and interquartile ranges. The differences between the re-positive and negative groups were compared with the Mann-Whitney test. Dichotomous or nominal categorical variables were compared with the chi-square test with normal approximation or Fisher's exact test, as appropriate. Some key clinical parameters that presented significant differences between the re-positive and negative groups were observed after the comparison. A binary logistic regression model (backward) was used to select the predictors of re-positive results according to the key clinical parameters. The predictive model for each key parameter was assessed based on the area under the receiver operating characteristic (ROC) curve (AUC). The 95\%
CI for each AUC was computed. Calibration of the final model was assessed graphically with a calibration plot. Twotailed $\mathrm{P}$ values less than 0.05 were considered statistically significant. Statistical analyses were performed with SPSS statistical software (IBM ${ }^{\circledR}$ SPSS Inc., Chicago, IL, USA) and the $\mathrm{R}$ programming language (version 3.5.1, R Foundation for Statistical Computing, Vienna, Austria).

\section{Results}

A total of 93 patients with COVID-19 were hospitalized in the Nanjing Public Health Medical Center. Three patients for whom key information was unavailable in the medical records were excluded, and 90 patients were included in the analysis. No patients died during hospitalization, and all patients were ultimately discharged. The median age of the 90 patients was 49 (IQR 33-60) years, with a range of 10 months to 97 years; two patients were younger than 14 years, and among them, the 10 months old boy was positive with retested RT-PCR. The re-positive patients were younger than that of the negative patients, but the difference was not significant (40 vs. 49, $\mathrm{P}=0.286$ ) (Table 1). The re-positive patients had a higher percentage of mild condition cases than did the negative patients $(\mathrm{P}=0.039)$. Comorbidities were present in more than half of the patients; surgery history $(17.8 \%)$ was the most common comorbidity, followed by cardiovascular cerebrovascular diseases (15.6\%) and digestive system diseases (8.9\%). The median time from illness onset to admission was 4 [2-7] days, whereas the median incubation period was 7 [4-12] days. The median peak body temperature was $38.2^{\circ} \mathrm{C}(37.8-38.9)$. The median threshold cycle of RNAemia was 30 [26-34]. The median duration of RT-PCR positivity was 10 days [5-13]. There were no significant differences in the incubation period, maximum body temperature, threshold cycle of RNAemia and number of RT-PCR positive days between the two groups (Table 1).

The most common symptom pre-admission was fever (73.3\%), followed by dry cough $(37.8 \%)$, cough and expectoration (20\%), and shortness of breath $(18.9 \%)$ (Table 2). After admission, patients showed new signs and symptoms; the most frequently observed post-admission complications were abdominal pain and diarrhea (40\%), followed by nausea and vomiting (22.2\%) and shortness of breath $(20 \%)$. The re-positive patients had a lower percentage of fever pre-admission ( $40 \%$ vs. $77.5 \%, \mathrm{P}=0.02)$ and a higher percentage of fever post-admission than negative patients $(30 \%$ vs. $6.3 \%, \mathrm{P}=0.042)$. There were no 
Table 1 Demographics and baseline characteristics

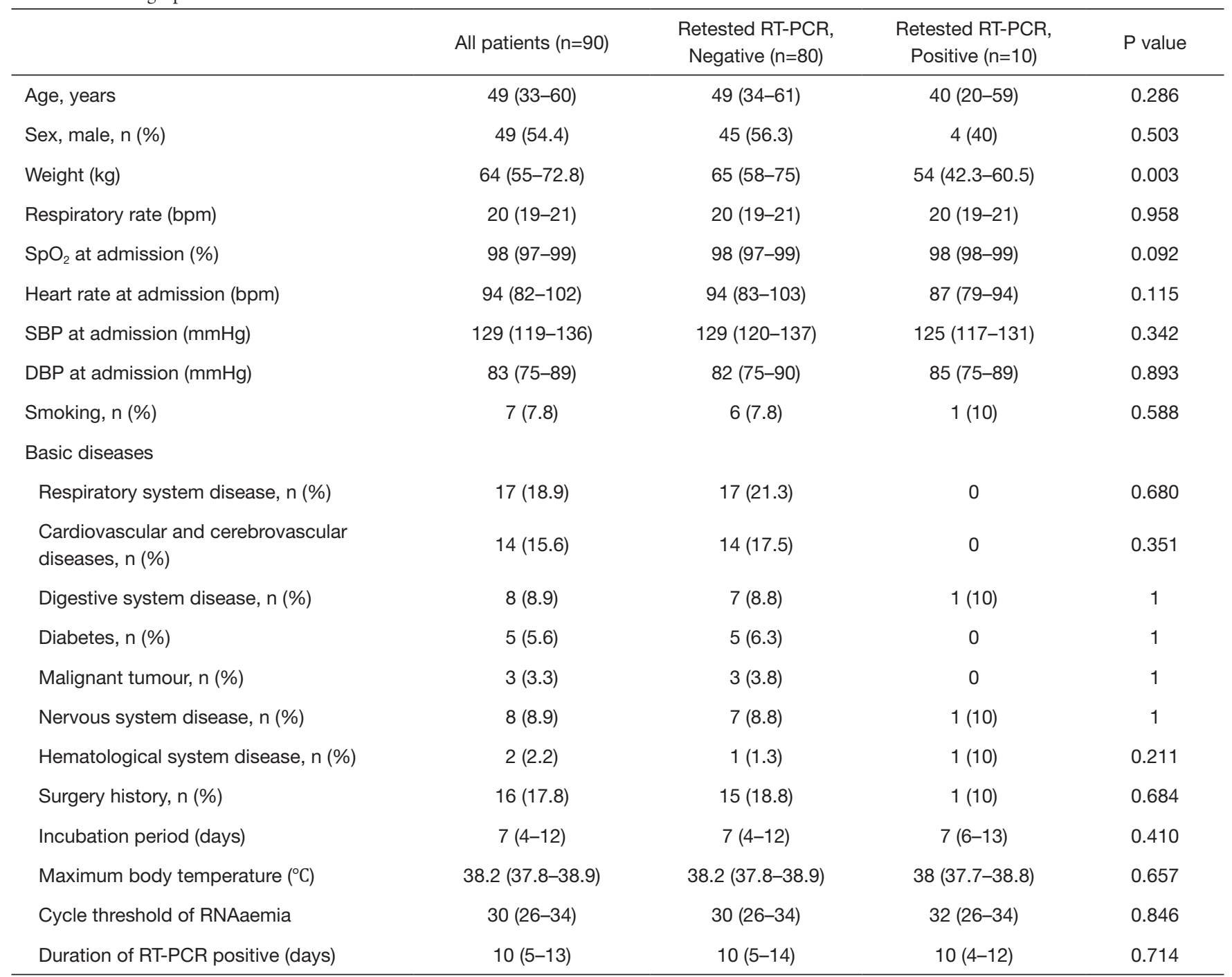

Data given as median (interquartile range, IQR) or percent of patients. Wilcoxon tests (continuous variables) and Fisher exact tests (categorical variables) were used to compare between retested RT-PCR positive and negative. $\mathrm{SpO}_{2}$, saturation of pulse oxygen; SBP, systolic blood pressure; DBP, diastolic blood pressure.

significant differences between the two groups in the other symptoms (Table 2).

Twenty-five (27.8\%) patients received antibiotics, and all patients received at least one antiviral medication. Thirtythree $(36.7 \%)$ patients received systematic glucocorticoids, and $44(48.9 \%)$ patients received intravenous immunoglobulin therapy (Table 3). There was no significant difference in medical treatment between the re-positive and negative patients.

The re-positive patients had higher WBC counts and creatine phosphokinase, and lower hs-CRP, IL-6 and ESR (all $\mathrm{P}<0.05$ ). There were no significant differences between the two groups in the other blood test parameters. Detailed laboratory findings are shown in Table 4.

We used three basic parameters (sex, age and weight) and eight clinical parameters (fever pre-admission, fever post-admission, unilateral lung infection, hs-CRP, WBC, creatine phosphokinase, interleukin-6 and ESR), which significantly differed between the re-positive and negative group, to model the independent risk of re-positivity by using logistic regression analysis (backward). Indicated that lower hs-CRP and higher WBC were independent risk 
Table 2 Signs and symptoms pre- and post-admission

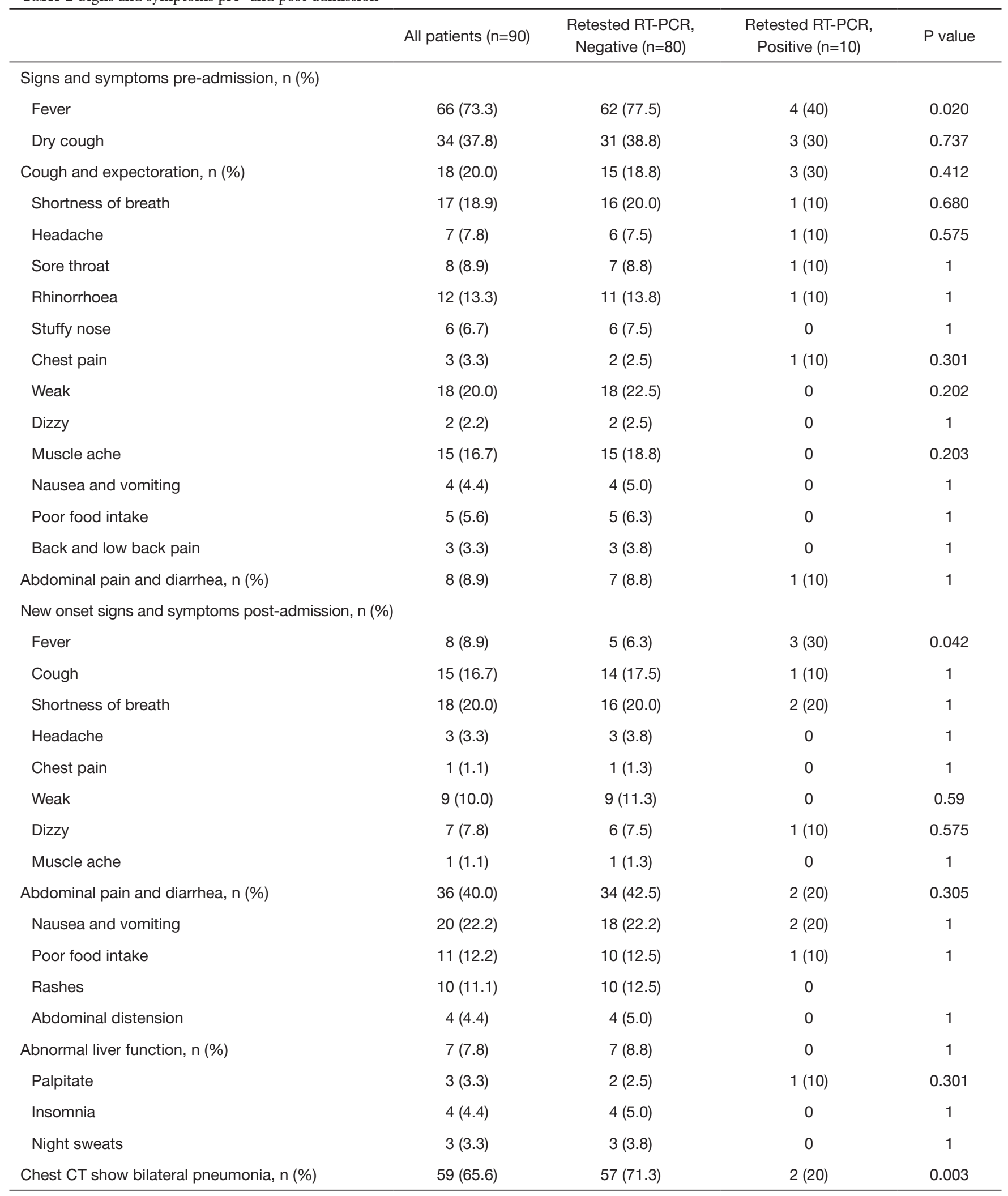

Data given as percent of patients. Fisher exact tests were used to compare between retested RT-PCR positive and negative. 
Table 3 Treatment of patients with COVID-19

\begin{tabular}{|c|c|c|c|c|}
\hline Treatment & All patients $(n=90)$ & Retested RT-PCR, Negative $(n=80)$ & Retested RT-PCR, Positive $(n=10)$ & $P$ value \\
\hline Glucocorticoids, n (\%) & $33(36.7)$ & $31(38.8)$ & $2(25.0)$ & 0.705 \\
\hline Immunoglobulin, n (\%) & $44(48.9)$ & $41(51.3)$ & $3(37.5)$ & 0.713 \\
\hline Albumin, n (\%) & $12(13.3)$ & $12(15.0)$ & 0 & 0.592 \\
\hline \multicolumn{5}{|l|}{ Anti-virus, n (\%) } \\
\hline Arbidol hydrochloride & $43(47.8)$ & $41(51.3)$ & $2(25.0)$ & 0.267 \\
\hline Lopinavir/ritonavir & $70(77.8)$ & $63(78.8)$ & $7(87.5)$ & 1 \\
\hline Darunavir/cobicistat & $31(34.4)$ & $28(35.0)$ & $3(37.5)$ & 1 \\
\hline
\end{tabular}

Data given as percent of patients. Fisher exact tests were used to compare between retested RT-PCR positive and negative. COVID-19, coronavirus disease 2019; RT-PCR, real-time reverse-transcriptase-polymerasechain-reaction.

Table 4 Laboratory findings of patients with COVID-19

\begin{tabular}{|c|c|c|c|c|}
\hline Laboratory findings & All patients $(n=90)$ & $\begin{array}{l}\text { Retested RT-PCR, } \\
\text { Negative }(n=80)\end{array}$ & $\begin{array}{l}\text { Retested RT-PCR, } \\
\text { Positive }(n=10)\end{array}$ & $P$ value \\
\hline $\mathrm{PaO}_{2} / \mathrm{FiO}_{2}$ ratio & $452.4(357.5-527.0)$ & $438.1(357.1-530.1)$ & $495.2(454.8-552.4)$ & 0.271 \\
\hline $\mathrm{PaCO}_{2}(\mathrm{mmHg})$ & $38(36-42)$ & $38(36-43)$ & $35(32-43)$ & 0.307 \\
\hline $\mathrm{PaO}_{2}(\mathrm{mmHg})$ & $103(86-118)$ & $102(84-119)$ & $104(96-116)$ & 0.605 \\
\hline Hs-CRP (mg/L) & $8.6(1.6-20)$ & $10.0(2.5-20)$ & $2.2(0.8-4.3)$ & 0.003 \\
\hline WBC $\left(10^{9} / \mathrm{L}\right)$ & $4.36(3.6-5.26)$ & $4.3(3.44-5.19)$ & $4.73(4.08-7.22)$ & 0.050 \\
\hline Absolute value of neutrophils $\left(10^{\circ} / L\right)$ & $2.61(1.90-3.14)$ & $2.53(1.88-3.15)$ & $2.64(2.19-3.28)$ & 0.663 \\
\hline Albumin (g/L) & $44.0(41.6-46.0)$ & $44.0(41.2-46.3)$ & $44.1(42.2-45.2)$ & 0.908 \\
\hline Total bilirubin (umol/L) & $10.6(8.0-14.2)$ & $10.8(8.5-14.8)$ & $8.2(5.7-11.3)$ & 0.063 \\
\hline AST (IU/L) & $21.9(18.4-33.2)$ & $21.9(18.4-35.1)$ & $21.8(16.5-27.3)$ & 0.555 \\
\hline $\operatorname{ALT}(\mathrm{U} / \mathrm{L})$ & $20.5(13.9-31.0)$ & $20.6(14.3-31.1)$ & $15.5(11.9-30.7)$ & 0.304 \\
\hline BUN (mmol/L) & $3.4(2.6-4.1)$ & $3.4(2.7-4.1)$ & $3.3(2.3-4.1)$ & 0.599 \\
\hline Creatinine $(\mu \mathrm{mol} / \mathrm{L})$ & $60(47-70)$ & $61.5(48-70)$ & $49(41-63)$ & 0.135 \\
\hline Venous blood Lactic acid (mmol/L) & $2.9(2.6-3.2)$ & $2.9(2.6-3.2)$ & $2.7(2.0-3.1)$ & 0.169 \\
\hline
\end{tabular}

Table 4 (continued) 
Table 4 (continued)

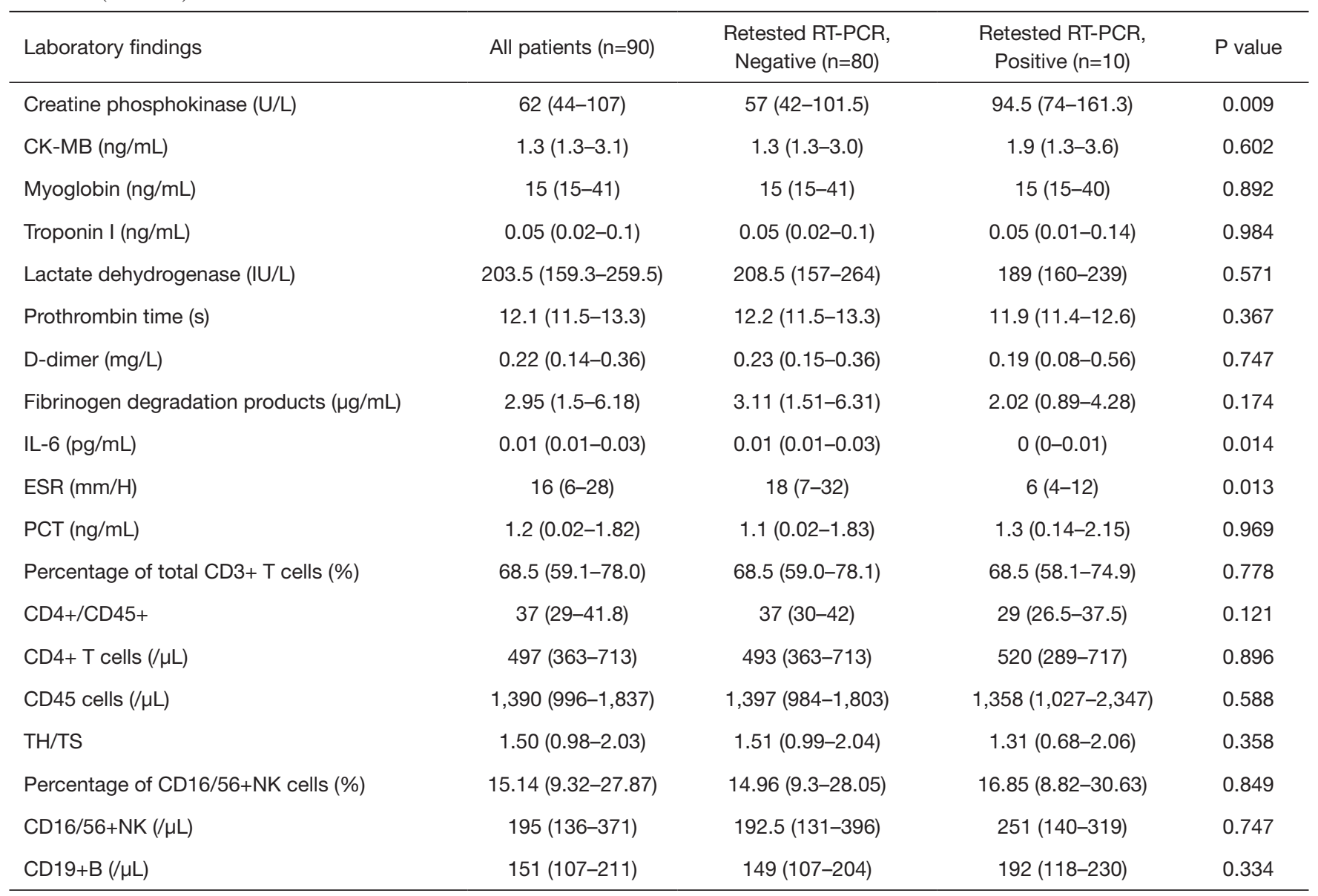

Data given as median (interquartile range) of patients. Wilcoxon tests were used to compare between retested RT-PCR positive and negative. COVID-19, coronavirus disease 2019; $\mathrm{PaO}_{2}$, partial pressure of oxygen; $\mathrm{FiO}_{2}$, fraction of inspiration oxygen; $\mathrm{pH}$, hydrogen ion concentration; $\mathrm{PaCO}_{2}$, partial pressure of carbon dioxide; hs-CRP, hypersensitive c-reactive protein; WBC, white blood cells; ALT, alanine aminotransferase; AST, aspartate aminotransferase; BUN, blood urea nitrogen; CK-MB, Creatine Kinase; IL-6, interleukin-6; ESR, erythrocyte sediment rate; PCT, procalcitonin; CD, cluster differentiation; TH, helper T; TS, suppressor T.

Table 5 Risk factors for re-positive

\begin{tabular}{lcccc}
\hline & Adjusted $\beta$ & P value & \multicolumn{2}{c}{$95 \%$ confidence interval } \\
\cline { 4 - 5 } Hs-CRP & & 0.049 & Lower & 0.999 \\
WBC & 1.96 & 0.025 & 1.088 & 3.640 \\
\hline
\end{tabular}

A binary logistic regression model (backward) was used to select the independent predictors of re-positive from eight key clinical parameters (pre-admission fever, post-admission fever, bilateral lung infection, hs-CRP, WBC, creatine phosphokinase, IL-6 and ESR). Abbreviations are defined in Table 4. 

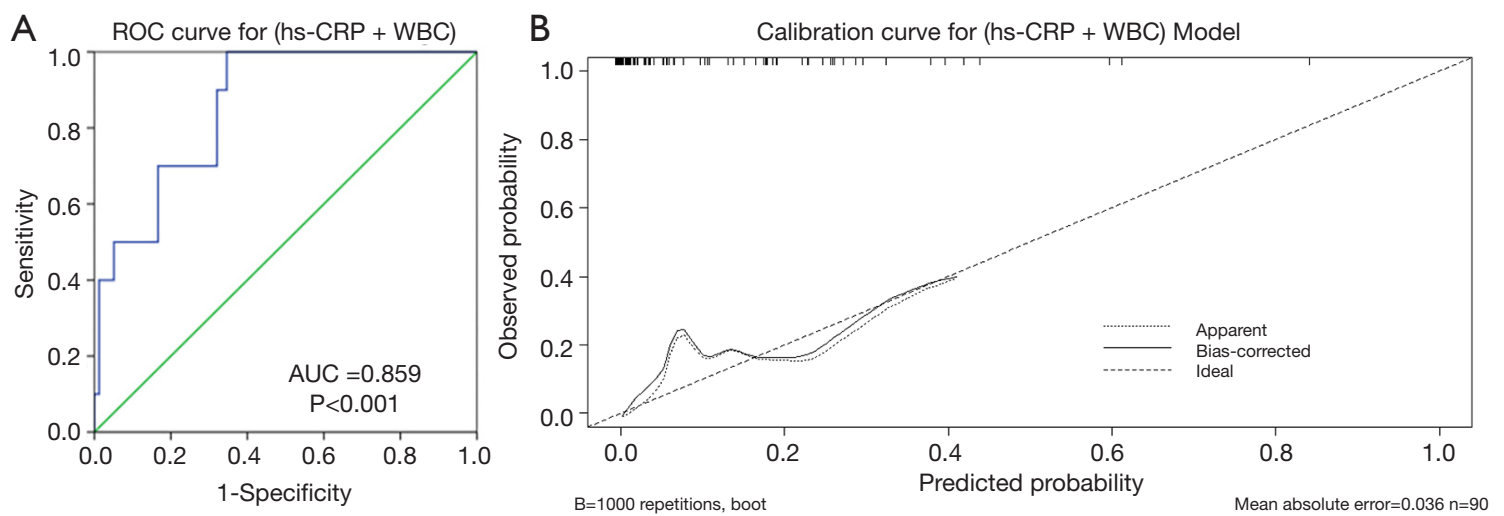

Figure 1 ROC curves for predictive models of RT-PCR re-positive testing based on hs-CRP + WBC together (A) and calibration curve for the hs-CRP + WBC model (B). ROC, receiver operator characteristic; AUC, area under the ROC curve; hs-CRP, hypersensitive c-reactive protein; WBC, white blood cells. RT-PCR, real-time reverse-transcriptase-polymerasechain-reaction.

factors for retesting positive (Table 5). These two parameters together had good predictive value for retesting positive, with an AUC of 0.859 (Figure 1A). Calibration curves showed that two models performed well across the spectrum of predicted probabilities of retesting positive (Figure 1B), with Hosmer-Lemeshow test $\mathrm{P}>0.5$.

\section{Discussion}

We provide the first reported study of the prevalence, characteristics and predictors of re-positive patients 4-20 days after therapy. Several case reports have described the features of re-positive patients, thus raising our concerns regarding RT-PCR re-positivity for SARS-CoV-2 (7-9). These studies have suggested that additional RTPCR testing prior to discharge should be performed. In our population, the youngest patient was 10 months old, and the oldest was 97 years old. Two patients were younger than 14 years, and among them, the 10 months old boy was positive with retested RT-PCR. We observed that people in all age groups are susceptible to infected by SARS-CoV-2, and it seems that the younger, the more likely to be repositive, although there was no significant difference of age between re-positive and negative groups. Wong et al. (10) have followed up 106 patients with nasopharyngeal swab testing and found that $21(19.8 \%)$ were re-positive. Their results support that an age above 60 years is a risk factor for SARS-CoV-2re-positivity. Deng and colleges (11) have reported that 61 of 576 observed patients (10.6) were repositive 3-35 days after discharge from the hospital. In our population, we observed $10(11 \%)$ pre-positive patients 4-20 days after therapy, findings similar to those in Deng's study. In our population, we investigated $10(11 \%)$ prepositive patients 4-20 days after therapy, which was similar to Deng's findings. We compared the clinical characteristics of these patients to those of the negative patients, and we found that the re-positive patients had a lower percentage of pre-admission fever, a higher percentage of post-admission fever, a lower percentage of bilateral lung infection, higher WBC count and creatine phosphokinase, and lower hsCRP, IL-6 and ESR (all $\mathrm{P}<0.05$ ). Our data indicated that the re-positive patients had milder symptoms and better blood testing results than the negative patients.

The two groups of patients had a similar threshold cycle for RNAemia, defined as the number of cycles required for the fluorescent signal to cross the threshold. These results suggested that the re-positive and negative patients had the same viral load when they were diagnosed. Yao XH has performed digital PCR on tissue sections from the lung, liver, heart, intestine, and skin of patients who died from COVID-19, and has unexpectedly found positive SARS$\mathrm{CoV}-2$ viral nucleic acid in only the lung but not other tissues (12). We speculated that the anti-viral and interferon treatment might have inhibited viral replication during hospital therapy, and that the virus in the re-positive group might have been more resistant to drugs and more likely to replicate after treatment ended. However, this viral replication did not lead to severe symptoms. Crucially, we believe that isolation for 20 days after the conclusion of therapy and retesting for SARS-CoV-2 would interrupt transmission and identify possible relapse.

Zhou et al. (13) and colleagues have suggested that re-positive patients are older and have poorer immune function and more underlying disease. However, our 
findings showed a different portrait in which re-positive patients were younger, and have mild clinical conditions and better blood testing results. Xie et al. (14) have reported positive ratio of nucleic acid detection for SARS-CoV-2 was $47.4 \%(9 / 19)$ in the 19 suspect patients. They speculated that infected patients could be missed by using nucleic acid detection only. Lei et al. (15) also considered that RTPCR was not always positive in patients with COVID-19. The authors have suggested making a diagnosis combining the CT scans and RT-PCR testing. Wang et al. (16) have collected 1,070 specimens from 205 patients with COVID-19 and have found that bronchoalveolar lavage fluid specimens showed the highest positive rates (14 of 15; $93 \%$ ), followed by sputum (72 of 104; $72 \%$ ), nasal swabs (5 of $8 ; 63 \%$ ), fibrobronchoscope brush biopsy (6 of 13; 46\%), pharyngeal swabs (126 of $398 ; 32 \%)$ and feces (44 of 153 ; $29 \%)$. Although feces can be used for RT-PCR detection of SARS-CoV-2, it is not a good method for clinical practice because of its lower positive rate. Because no person-toperson transmission was observed, we suggest isolation for more than 14 days after the ending of treatment and subsequent retesting of sputum rather than adding other testing procedures.

Several studies have mentioned WBC, most of which have found that patients with severe disease have higher WBC counts than those with non-severe disease $(3,17$ 20). A recent study has compared WBC counts between COVID-19-positive and COVID-19-negative individuals and found that the former had lower WBC counts and higher CRP levels, contrary to our findings (21). However, no report has described the role of WBC in re-positive patients. Huang et al. (3) have found that ICU patients have higher WBC, neutrophil count and bilirubin, and lower lymphocyte counts than non-ICU patients. Zhou et al. (17) have reported that non-survivors have lower platelet counts and albumin, and higher ALT and IL-6, than survivors. In the present study, the re-positive patients had lower IL-6 and ESR than the negative patients, thus also reflecting that the re-positive patients were not as severe as those of the negative patients.

Liu et al. (22) have found that age, viral load, lung injury score, blood biochemistry indexes, albumin, CRP, lactate dehydrogenase, lymphocytes (\%), and lymphocytes and neutrophils (\%) may be predictors of disease severity in a 12-case study. Mo et al. (23) have reported that male sex, anorexia and no fever on admission are predictive of poor efficacy. The current results suggest that lower hs-CRP and higher WBC are independent risk factors for retesting positive. However, in this study, we sought to predict a different outcome from those in previous studies, and our specific results are different from the results of those studies. In this study, the combination of hs-CRP and WBC had good performance for predicting retesting positive, with an AUC of 0.859. Although the clinical indicators had a good predictive value, we consider these results preliminary. However, our findings suggest that more attention should be paid to the common clinical phenomenon of retesting positive by RT-PCR for SARS-CoV-2.

There are several limitations in the current study. First, the re-positive diagnosis depend on a repeat RTPCR, which maybe false-negative. So, we consider that the realistic percentage of relapse patients may be higher than our observation. Second, according to our statistical results, most of the re-positive patients were mild patients infected with novel coronavirus. They may have low viral load, weak immune response and long virus carrying time. Third, because at least 14 days of isolation and medical observation occurred post-therapy, we did not observe any person-to-person transmission for the re-positive patients. However, we cannot conclude that there was no infection among the re-positive patients. Last but not least, although we report the first study focusing on re-positive patients, this was a single-center, small sample study, thus potentially producing bias. Further large sample, multicenter studies must be performed.

\section{Conclusions}

We conclusion that $11 \%$ (10) patients retested positive by RT-PCR for SARS-CoV-2 4-20 days after completion of therapy and these patients had mild symptoms and better blood testing results than those retested negative patients. A combination of hs-CRP and WBC, with an AUC of 0.859, may predict positive retesting by RT-PCR; however, the sensitivity and specificity should be studied further.

\section{Acknowledgments}

We appreciate all the patients for their informed consent and all the health care workers involved in the fight against COVID-19.

Funding: This work was supported by 511 Take-off Plan [grant number: JSPH-511C-2018-10] and the 2018 Annual general project of Nanjing Medical Science and Technology Development Funding [grant number: YKK18153]. The sponsor had no role in the design of the study, the 
collection and analysis of the data, or the preparation of the manuscript.

\section{Footnote}

Data Sharing Statement: Available at http://dx.doi. org/10.21037/jtd-20-1547

Conflicts of Interest: All authors have completed the ICMJE uniform disclosure form (available at http://dx.doi. org/10.21037/jtd-20-1547). All authors have no conflicts of interest to declare.

Ethical Statement: The authors are accountable for all aspects of the work in ensuring that questions related to the accuracy or integrity of any part of the work are appropriately investigated and resolved. The study was conducted in accordance with the Declaration of Helsinki (as revised in 2013). This case series was approved by the Institutional Ethics Board of the Second Hospital of Nanjing (No. 2020-L-S-ky003). Oral consent was obtained from patients.

Open Access Statement: This is an Open Access article distributed in accordance with the Creative Commons Attribution-NonCommercial-NoDerivs 4.0 International License (CC BY-NC-ND 4.0), which permits the noncommercial replication and distribution of the article with the strict proviso that no changes or edits are made and the original work is properly cited (including links to both the formal publication through the relevant DOI and the license). See: https://creativecommons.org/licenses/by-nc-nd/4.0/.

\section{References}

1. Hui DS, I Azhar E, Madani TA, et al. The continuing $2019 \mathrm{nCoV}$ epidemic threat of novel coronaviruses to global health the latest 2019 novel coronavirus outbreak in Wuhan, China. Int J Infect Dis 2020;91:264-6.

2. Lu H, Stratton CW, Tang YW. Outbreak of pneumonia of unknown etiology in Wuhan, China: The mystery and the miracle. J Med Virol 2020;92:401-2.

3. Huang C, Wang Y, Li X, et al. Clinical features of patients infected with 2019 novel coronavirus in Wuhan, China. Lancet 2020;395:497-506.

4. Singhal T. A Review of Coronavirus Disease-2019 (COVID-19). Indian J Pediatr 2020;87:281-6.

5. Wu Z, McGoogan JM. Characteristics of and Important
Lessons From the Coronavirus Disease 2019 (COVID-19)

Outbreak in China: Summary of a Report of 72,314

Cases From the Chinese Center for Disease Control and Prevention. JAMA 2020;323:1239-42.

6. Guan WJ, Ni ZY, Hu Y, et al. Clinical Characteristics of Coronavirus Disease 2019 in China. N Engl J Med 2020;382:1708-20.

7. Lan L, Xu D, Ye G, et al. Positive RT-PCR Test Results in Patients Recovered From COVID-19. JAMA 2020;323:1502-3.

8. Cao H, Ruan L, Liu J, et al. The clinical characteristic of eight patients of COVID-19 with positive RT-PCR test after discharge. J Med Virol 2020;10.1002/jmv.26017. [Epub ahead of print].

9. Chen Y, Bai W, Liu B, et al. Re-evaluation of retested nucleic acid-positive cases in recovered COVID-19 patients: Report from a designated transfer hospital in Chongqing, China. J Infect Public Health 2020;13:932-4.

10. Wong J, Koh WC, Momin RN, et al. Probable causes and risk factors for positive SARS-CoV-2 test in recovered patients: Evidence from Brunei Darussalam. J Med Virol 2020;10.1002/jmv.26199. [Epub ahead of print].

11. Deng W, Guang TW, Yang M, et al. Positive results for patients with COVID-19 discharged form hospital in Chongqing, China. BMC Infect Dis 2020;20:429.

12. Yao XH, He ZC, Li TY, et al. Pathological evidence for residual SARS-CoV-2 in pulmonary tissues of a ready-fordischarge patient. Cell Res 2020;30:541-3.

13. Zhou L, Liu K, Liu HG. Cause analysis and treatment strategies of "recurrence" with novel coronavirus pneumonia (COVID-19) patients after discharge from hospital. Zhonghua Jie He He Hu Xi Za Zhi 2020;43:281-4.

14. Xie C, Jiang L, Huang G, et al. Comparison of different samples for 2019 novel coronavirus detection by nucleic acid amplification tests. Int J Infect Dis 2020;93:264-7.

15. Lei P, Fan B, Mao J, Wang P. Comprehensive analysis for diagnosis of novel coronavirus disease (COVID-19) infection. J Infect 2020. [Epub ahead of print].

16. Wang W, Xu Y, Gao R, et al. Detection of SARSCoV-2 in Different Types of Clinical Specimens. JAMA 2020;323:1843-4.

17. Zhou F, Yu T, Du R, et al. Clinical course and risk factors for mortality of adult inpatients with COVID-19 in Wuhan, China: a retrospective cohort study. Lancet 2020;395:1054-62.

18. Gong J, Dong H, Xia SQ, et al. Correlation Analysis Between Disease Severity and Inflammation-related 
Parameters in Patients With COVID-19 Pneumonia. medRxiv 2020:2020.02.25.20025643.

19. Qin C, Zhou L, Hu Z, et al. Dysregulation of Immune Response in Patients With Coronavirus 2019 (COVID-19) in Wuhan, China. Clin Infect Dis 2020;71:762-8.

20. Wang D, Hu B, Hu C, et al. Clinical characteristics of 138 hospitalized patients with 2019 novel coronavirus-infected pneumonia in Wuhan, China. JAMA 2020;323:1061-9.

21. Ferrari D, Motta A, Strollo M, Banfi G, Locatelli M.

Cite this article as: Liu Y, Ding N, Zhou S, Chen C, Huang S, Lv Y, Jiao D, Zheng Y, Hu Z, Xu C, Chen W, Dai H, Sun W, Cheng C, Lv R, Cheng J, Ye Z, Li Z, Yi Y, Wei H. Comparison of clinical characteristics between patients with coronavirus disease 2019 (COVID-19) who retested RT-PCR positive versus negative: a retrospective study of data from Nanjing. J Thorac Dis 2020;12(11):6435-6445. doi: 10.21037/jtd.2020.04.17
Routine blood tests as a potential diagnostic tool for COVID-19. Clin Chem Lab Med 2020;58:1095-9.

22. Liu Y, Yang Y, Zhang C, et al. Clinical and biochemical indexes from 2019-nCoV infected patients linked to viral loads and lung injury. Sci China Life Sci 2020;63:364-74.

23. Mo P, Xing Y, Xiao Y, et al. Clinical characteristics of refractory COVID-19 pneumonia in Wuhan, China. Clin Infect Dis 2020. [Epub ahead of print]. 